\title{
Anesthetic Management of a Patient with Myasthenia Gravis for Meningioma Surgery - A Case Report
} Srivastava V K, ${ }^{1}$ Agrawal $A,{ }^{2}$ Ahmed $M,{ }^{1}$ Sharma $\mathrm{S}^{1}$

\author{
${ }^{1}$ Apollo Hospitals Bilaspur, \\ Chhattisgarh, India \\ ${ }^{2}$ Himalayan Institute of Medical Sciences, \\ Dehradun, India
}

Corresponding Author

Vinit K. Srivastava

Department of Anaesthesia

Apollo Hospitals Bilaspur,

Chhattisgarh, India 495006

Email: drvinit75@gmail.com

Citation

Srivastava V K, Agrawal A, Ahmed M, Sharma S. Anesthetic Management of a Patient with Myasthenia Gravis for Meningioma Surgery - A Case Report. Kathmandu Univ Med J 2015;49(1):80-2.

\begin{abstract}
Myasthenia gravis is a disease of great challenge to the anesthesiologist, because it affects the neuromuscular junction. Anesthetic management involves either muscle relaxant or non-muscle relaxant techniques. This case report documents the safe use of fentanyl, propofol and sevoflurane combination guided by bispectral index, without the use of muscle relaxants in a patient with myasthenia gravis who presented for meningioma surgery.
\end{abstract}

\section{KEY WORDS}

Fentanyl, myasthenia gravis, propofol, sevoflurane

\section{INTRODUCTION}

Myasthenia Gravis (MG) is an autoimmune disease characterized by weakness and fatigability of skeletal muscles, with improvement following rest. Ptosis and diplopia occur early in the majority of patients. Myasthenia Gravis is caused by production of antibodies against the acetylcholine receptor (anti-AchR) resulting in a reduction of the number of functional receptors at the neuromuscular synapse. ${ }^{1}$ Therefore, careful perioperative management is required because of the unpredictable susceptibility to analgesia and muscle relaxants. ${ }^{2}$ It may be associated with other disorders of autoimmune origin, such as thyroid hypofunction, rheumatoid arthritis, systemic lupus erythematosus and diabetes mellitus. To date, there is one case report, which describes the perioperative management of patients with myasthenia gravis undergoing neurosurgery. ${ }^{3}$

Anesthesia for neurosurgery in MG is challenging. In this case report, we describe the successful perioperative management of a patient with myasthenia gravis who underwent craniotomy and decompression of tumor. We have adopted a non-muscle relaxant anesthetic technique which is widely used nowadays.

\section{CASE-REPORT}

A 62 year old female, weighing $60 \mathrm{~kg}$ presented with complaints of sudden onset of giddiness and left sided weakness for last three days. She was a known case of myasthenia gravis for last eight years, control on tab pyridostigmine $60 \mathrm{mg}$ T.D.S. and tab azathioprine $50 \mathrm{mg}$ O.D. She was also a known case of diabetes mellitus and hypertension for last five years, control on oral hypoglycemic agents and tab. telmisartan amlodipine combination. She was diagnosed as a case of right frontoparietal meningioma and scheduled for craniotomy and tumor decompression. All routine investigations were in normal limits. Written informed high risk consent and consent for postoperative ventilator support was taken.

The patient was premedicated with tab. ranitidine $150 \mathrm{mg}$ night before and two hours prior to surgery. No preoperative sedative medication was given. The patient was continued on the routine schedule of pyridostigmine until the morning of surgery. In the operating room, standard monitoring, including ECG, pulse oximetry and noninvasive blood pressure were attached. Electrodes were applied to the patient's forehead for monitoring the bispectral index (BIS) of the electroencephalogram. Peripheral venous, left radial 
artery and right internal jugular vein cannulation were performed under local anesthesia. Her preinduction heart rate was 78 beats per minute, arterial blood pressure (ABP) was 138/80 $\mathrm{mmHg}$ and central venous pressure (CVP) was $4 \mathrm{mmHg}$. Preoperatively nebulisation was done with $4 \mathrm{ml}$ of $4 \%$ xylocaine for 15 minutes then gargle with viscous xylocaine $4 \%$. Larynx was sprayed with $10 \%$ xylocaine.

Anesthesia was induced with injection (inj.) glycopyrrolate $0.2 \mathrm{mg}$, midazolam $0.03 \mathrm{mg} / \mathrm{kg}$, fentanyl $2 \mu \mathrm{g} / \mathrm{kg}$ and propofol $3 \mathrm{mg} / \mathrm{kg}$ body weight. At the loss of eye-lash reflex, an oral airway was placed and the lungs were manually hyperventilated (end tidal $\mathrm{CO}_{2} 25-30 \mathrm{mmHg}$ ) with the sevoflurane gas mixture. After $1 \mathrm{~min}$ of induction $2 \mathrm{ml} 2 \%$ lidocaine was injected through the cricothyroid membrane to prevent coughing during intubation. Trachea was intubated with an appropriate sized cuffed endotracheal tube without using a neuromuscular blocking agent when the BIS value was 35. Maintenance of anesthesia was done with $\mathrm{O}_{2}: \mathrm{N}_{2} \mathrm{O}(40: 60)$, sevoflurane, propofol infusion and intermittent boluses of fentanyl. The patient was ventilated mechanically to keep the end-tidal carbon dioxide tension within 28-30 mmHg. Doses of anesthetic agents were modified according to the clinical assessment of the depth of anaesthesia (BIS value 40-45). The temperature was maintained at around $36^{\circ} \mathrm{C}$.

Tumor decompression was not possible because it was surrounded by thick veins so only excision biopsy was taken. Surgery was lasted for 3 hours. The patient's intraoperative course was uneventful, with the heart rate remaining between 72 and 94 beats/ min, blood pressure 140-96/86$62 \mathrm{~mm} \mathrm{Hg}$. The volume replacement was guided by CVP and the patient received $1500 \mathrm{~mL}$ of normal saline only. The total blood loss was $100 \mathrm{ml}$ and urine output $200 \mathrm{ml}$. She did not require blood transfusion. Ten minutes after stopping the anesthetics, patient was able to open her eyes, and breath spontaneously and generating adequate tidal volume, BIS value was increased to 84 . Trachea was extubated and she responded to verbal commands at 5th min. after extubation. Her hemodynamic parameters were stable postoperatively. Postoperative pain relief was continued with fentanyl infusion $20 \mu \mathrm{g} / \mathrm{hr}$ and paracetamol 1 gm i.v. 8 hrly. Tab pyridostigmine $60 \mathrm{mg}$ T.D.S. was reintroduced after 6 hours of surgery in the postoperative period. The patient was discharged from the hospital on $7^{\text {th }}$ postoperative day.

\section{DISCUSSION}

Myasthenia gravis patients, particularly those undergoing major surgery require special anaesthesia technique, surgical preparation and postoperative care. ${ }^{4}$ Myasthenic patients may have little respiratory reserve hence depressant drugs for preoperative premedication should be used with caution, and avoided in patients with bulbar symptoms, but an anticholinergic may be useful.
Studies have suggested that the optimization of the medical condition with pyridostigmine until the morning of surgery followed by anesthesia using isoflurane with one-tenth the standard intubating dose of vecuronium in patients with myasthenia gravis is safe. ${ }^{5}$ The omission of pyridostigmine on the night before surgery makes patients more susceptible to develop muscle weakness while waiting for the surgery and the patients show significant sensitivity towards vecuronium. So we continued the morning dose of pyridostigmine to prevent any muscle weakness.

Anesthetic goals include smooth induction, stable hemodynamic course, rapid emergence and early extubation for neurological examination in patients with myasthenia gravis undergoing neurosurgical procedure.

In the past, many different anesthetic techniques have been used. Fentanyl-propofol combination, fentanyl-sevoflurane combination, and more recently, a sufentanyl-propofol or remifentanil-propofol combination have been reported in the literature with varying results..$^{6-8}$ Traditionally, great reliance has been placed on the volatile anesthetic agents for induction and maintenance of anesthesia in MG patients. These agents may be associated with complications like cough, laryngospasm, breath holding and delayed recovery after the anesthesia. Sevoflurane has low blood/gas and tissue/gas solubility and may be a suitable volatile anesthetic agent for general anesthesia in patients with MG. Sevoflurane also has an inhibitory effect on neuromuscular transmission in MG patients. ${ }^{9}$

We used propofol with sevoflurane combination, that provide good intubating conditions without the need for muscle relaxants and adequate depth of anesthesia (BIS 35 at the time of intubation), which was very much required in our patient. ${ }^{10}$ This was supplemented with local anesthesia of airway to prevent marked change in haemodynamic parameters and change of intracranial pressure during intubation and maintenance phase.

Propofol has the advantages of short duration of action without effect on neuromuscular transmission. Opioid analgesics in therapeutic concentrations do not appear to depress neuromuscular transmission in myasthenic muscle. ${ }^{11}$ However, central respiratory depression may be a problem with opioids. The introduction of shortacting opioids makes these drugs more titratable in the myasthenic. ${ }^{8}$ Remifentanil's short elimination half-life $(9.5$ minutes makes the drug appealing. But as ramifentanyl was not available that's why we have used fentanyl.

Patients of mysthenia gravis are known to be extremely sensitive to the effects of non-depolarizing muscle relaxants. The degree of sensitivity is variable even among patients who are in complete remission, although patients who have severe disease and require higher doses of pyridostigmine tend to be more sensitive than others. ${ }^{12}$ Hence, caution is required while using non-depolarizing agents in patients with myasthenia gravis. 
Baraka et al have reported that the effect of cis-atracurium is potentiated by concurrent use of sevoflurane. ${ }^{13}$ Considering the possible delayed recovery by use of muscle relaxant in the maintenance phase, we use propofol infusion, intermittent fentanyl and sevoflurane gas mixture for maintenance as guided by BIS value.

\section{REFERENCES}

1. Blichfeldt-Lauridsen L, Hansen BD. Anesthesia and myasthenia gravis. Acta Anaesthesiol Scand. 2012;56(1):17-22.

2. Drachman DB. Myasthenia gravis. N Engl J Med 1994;330(25):1797810.

3. Sanghavi N, Shelat S, Pattanayak D: Neurosurgery in a Symptomatic Myasthenia Gravis Patient. The Internet Journal of Anesthesiology. 2008 Volume 17 Number 1.

4. Cardone A, Congedo E, Aceto P, Sicuranza R, Chinè E, Caliandro F, et al. Perioperative evaluation of myasthenia gravis. Ann Ital Chir. 2007;78(5):359-65.

5. Tripathi M, Kaushik S, Dubey P. The effect of use of pyridostigmine and requirement of vecuronium in patients with myasthenia gravis. $J$ Postgrad Med. 2003;49(4):311-4.

6. Della Rocca G, Coccia C, Diana L, Pompei L, Costa MG, Tomaselli E, et al. Propofol or sevoflurane anesthesia without muscle relaxants allow the early extubation of myasthenic patients. Can J Anaesth. 2003;50(6):547-52.

7. El-Dawlatly AA. Anesthesia for thoracoscopic thymectomy: modified non-muscle relaxant technique--case reports. Middle East J Anesthesiol 2007;19(1):219-24.
In conclusion, we found that the avoidance of muscle relaxants and use of fentanyl, propofol and sevoflurane combination guided by BIS can provide a smooth induction, stable haemodynamic course, rapid emergence and early extubation in patients with myasthenia gravis undergoing neurosurgical procedure.

8. Ng JM. Total intravenous anesthesia with propofol and remifentanil for video-assisted thoracoscopic thymectomy in patients with myasthenia gravis. Anesth Analg 2006;103(1):256-7.

9. Nitahara K, Sugi Y, Higa K, Shono S, Hamada T. Neuromuscular effects of sevoflurane in myasthenia gravis patients. Br J Anaesth. 2007;98(3):337-41.

10. Maddali MM, Matreja PV, Zachariah M. Bispectral index-monitored anesthesia technique for transsternal thymectomy. Asian Cardiovasc Thorac Ann. 2009;17(4):389-94.

11. Sanders DB, Kim YI, Howard JF Jr, Johns TR, Muller WH Jr. Intercostal muscle biopsy studies in myasthenia gravis: clinical correlations and the direct effects of drugs and myasthenic serum. Ann $N$ Y Acad Sci. 1981;377:544-66.

12. Nilsson E, Meretoja OA. Vecuronium dose-response and maintenance requirements in patients with myasthenia gravis. Anesthesiology. 1990;73(1):28-32.

13. Baraka AS, Taha SK, Kawkabani NI. Neuromuscular interaction of sevoflurane--cisatracurium in a myasthenic patient. Can J Anaesth. 2000;47(6):562-5. 